\title{
Mathematical modeling of robust pattern formation in the Drosophila eye disc
}

\author{
Svetlana Surkova \\ SPbPU, St. Petersburg, Russia \\ surkova_syu@spbstu.ru \\ Sergey Nuzhdin \\ USC, Los Angeles, USA \\ snuzhdin@usc.edu
}

\author{
Vitaly Gursky \\ Ioffe Institute, St. Petersburg,Russia \\ gursky@math.ioffe.ru \\ Maria Samsonova \\ SPbPU, St. Petersburg, Russia \\ m.samsonova@spbstu.ru
}

\author{
Konstantin Kozlov \\ SPbPU, St. Petersburg, Russia \\ kozlov_kn@spbstu.ru
}

\begin{abstract}
We apply mathematical modeling approach to study mechanisms underlying the robust formation of periodic pattern during the Drosophila eye development. Model is fitted to the preliminary quantitative data - concentrations of the main regulators of $R 8$ cell selection and specification. We analyze how biological noise propagates within the model to explain the molecular mechanisms responsible for noise buffering.
\end{abstract}

Keywords - Drosophila eye imaginal disc, quantitative gene expression, mathematical modeling, robustness of development

\section{Motivation and Aim}

Morphogenesis of many biological structures is characterized by formation of periodic patterns from nearly homogeneous tissues. However, mechanisms underlying the robustness of such patterns are still not fully clarified. Specification of photoreceptor cells during eye development in Drosophila is an interesting example of rapidly forming highly ordered structure. This structure is initially determined by the precise spatial arrangement of R8 cells (presumptive photoreceptors R8) in the eye imaginal disc during larval stage of Drosophila development. Despite large amount of experimental data on molecular mechanisms of eye disc patterning, quantitative studies were published only recently. The expression levels of main regulators of R8 cell specification showed significant variation between Drosophila inbred lines, sexes and species [1]. This is unclear, how a precise structure of spatial distribution of R8 cells is formed despite variable levels of regulators, and what molecular mechanisms are involved in stabilization of this structure. To address these questions, we use mathematical modeling approach. The published modeling studies are formulated in terms of 'generalized' activators and repressors involved in $\mathrm{R} 8$ cell specification $[2,3]$. The objective of this study was to develop the approach where the mechanism of pattern formation is inferred as a consequence of the correct description of experimental data by the model.

\section{Methods}

The preliminary dataset used for model development included confocal images of Drosophila eye imaginal discs stained for the expression of atonal, hedgehog, hairy, Delta (Ali et al., 2019), Notch and cubitus interruptus. We used Hybridization Chain Reaction (HCR) technique for mRNA detection [4] and combined it with immunohistochemistry. The MrComas method was applied for quantification of gene expression from the confocal images [1]. Data on expression of other regulators of $\mathrm{R} 8$ cell selection and specification were obtained from literature.

\section{Results}

The model is based on the regulatory network which comprises the main factors and interactions between them relying on the available experimental information. However, the type of regulation (activation or repression) still needs to be clarified for some interactions. The model is formulated in terms of ordinary differential equations. Cell signaling is implemented in the model in two ways. First, transport of cell nonautonomous factors (e.g. Hedgehog) is described by the two-dimensional Laplacian operator, which is the diffusion operator discretized on the cell grid. Second, we include the Delta-Notch signaling mechanism in the model. We started with modification of the modeling approach implementing 'switch and template' pattern formation mechanism [2] and a generalized model with a short-range activator [3] by replacing the generalized activators and repressors with the real regulators of $\mathrm{R} 8$ cell specification. We fit this model to the quantitative expression data and analyze the resulted model behavior. The noise propagation in the model is estimated with the help of parameters characterizing the pattern regularity, which include the inhomogeneity index of spatial distances between the differentiated R8 cells, the inhomogeneity index of angles between photosensitive units (ommatidia), and the total number of ommatidia. We study perturbations of spatial positions of differentiating cells, as well as perturbations of parameter values, initial conditions, and disc geometry.

\section{ACKNOWLEDGMENT}

This study was supported by Russian Foundation for Basic Research (RFBR) grant 20-04-01047-a.

\section{REFERENCES}

[1] Ali S, Signor SA, Kozlov K, Nuzhdin SV. (2019). Novel approach to quantitative spatial gene expression uncovers genetic stochasticity in the developing Drosophila eye. Evol Dev. May;21(3):157-171.

[2] Lubensky DK, Pennington MW, Shraiman BI, Baker NE. (2011) A dynamical model of ommatidial crystal formation. Proc Natl Acad Sci USA. 108: 11145-11150.

[3] Gavish A, Shwartz A, Weizman A, Schejter E, Shilo B-Z, Barkai N. (2016) Periodic patterning of the Drosophila eye is stabilized by the diffusible activator Scabrous. Nat Commun. 7: 10461.

[4] Choi, H.M.T., Beck, V.A., Pierce, N.A. (2014) Next-generation in situ hybridization chain reaction: higher gain, lower cost, greater durability. ACS Nano 8: 4284-4294. 